\title{
V. AUTORAS Y AUTORES
}

\section{Octavio Salazar Benítez}

Profesor Titular de D. Constitucional de la Universidad de Córdoba. Responsable del Grupo de Investigación "Democracia, pluralismo y ciudadanía". Autor de Cartografias de la igualdad (Tirant lo Blanch, 2010) y Masculinidades y ciudadanía. Los hombres también tenemos género (Dykinson, 2013). Miembro de la Red Feminista de Derecho Constitucional.

\section{Nieves Alonso García}

Becaria de Doctorado en el Departamento de Derecho Constitucional de la Universidad de León. Licenciada en Ciencias Políticas y de la Administración por la Universidad de Salamanca, Máster en Asesoría Jurídica por la Universidad de León, finalizando Grado en Derecho en la Universidad de León.

\section{María Macías Jara}

Profesora Doctora de Derecho Constitucional en la Universidad Pontificia Comillas - ICADE. Autora de materiales y Responsable de la asignatura "Los Derechos de las Mujeres" del Máster de Derechos Humanos de la Universidad de Alcalá. Ha centrado su investigación en materias relativas a derechos fundamentales e igualdad de género, en particular, en el ámbito de la representación política, así como, entre otros, a la exigibilidad y garantía de los derechos sociales, temas sobre los que versan la mayor parte de sus publicaciones.

\section{Inmaculada Hurtado García}

Profesora Colaboradora Doctora. Universidad CEU-Cardenal Herrera. Doctora Europea en Antropología de la Medicina (Universidad Rovira i Virgili). Premio Extraordinario de Doctorado. Licenciada en Antropología Social y Cultural (Universidad Miguel Hernández). Primer Premio de Investigación Cultural Marqués de Lozoya (2010) Ministerio de Cultura.

\section{Elia Saneleuterio}

Doctora Europea (Literatura Española), licenciada en Filología Hispánica, maestra en Educación Infantil y máster en Investigación en Didácticas Específicas. Profesora de 
Magisterio en el Departament de Didàctica de la Llengua i la Literatura de la Universitat de València y autora de numerosos capítulos de libros, artículos de investigación y monografías.

\section{Rocío López-García-Torres}

Profesora del Departamento de Ciencias de la Educación de la Universidad CEU-Cardenal Herrera. Licenciada en Psicología y Doctora en Ciencias de la Educación por la Universidad de Granada.

\section{Elena Juaristi Besalduch}

Profesora del Departamento de Derecho Público de la Universidad CEU-Cardenal Herrera. Licenciada en Derecho por la Universitat de València. Máster en Agente de Igualdad de Oportunidades. Doctora Internacional en Derecho por la Universidad CEU-Cardenal Herrera

\section{María García-Alcober}

Profesora del Departamento de Economía y Empresa de la Universidad CEU-Cardenal Herrera. Doctora en Economía con mención internacional por la Universitat Jaume I. Licenciada en Economía por la Universitat de València.

\section{Ruth Abril Stoffels}

Doctora en Derecho, Licenciada en Derecho por la UCM y en Ciencias Políticas por la UNED. Máster Agentes de Igualdad UDL. Máster en Estudios Europeos (Colegio de Europa). Actualmente es profesora de Derecho Internacional y Derechos Humanos en la Universidad CEU-Cardenal Herrera, en la que dirige la Unidad de Igualdad.

\section{María Angustias Díaz Gómez}

Catedrática de Derecho Mercantil, Directora del Departamento de Derecho Privado y de la Empresa de la Universidad de León, ex Vocal del Tribunal de Defensa de la Competencia de Castilla y León.

\section{Elena F. Pérez Carrillo}

Doctora en Derecho, Profesora de Derecho Mercantil de la Universidad de León. 


\section{Helena Rodemann Rounsevell}

Máster en Intervención en Violencia contra las Mujeres, 2014-2015 por la Universidad de Deusto; Bachelor of Arts (B.A.) in English, National Honors Society and Cum Laude, 20062010 por Marymount Manhattan College; Locutora de radio, 2013-2014 en Sorginen Leizea, Irola Irratia; Redactora, 2013-2014 em Ecolocalizer.

\section{Claudia Jessenia Becerra Gualdrón}

Investigadora Universidad Pedagógica y Tecnológica de Colombia - Centro Regional de Gestión para la productividad y la Innovación de Boyacá. Av Central del Norte UPTC oficina C115 (Tunja, Boyacá, Colombia). Economista, candidata a Magíster en Economía de la Universidad Pedagógica y Tecnológica de Colombia UPTC, con experiencia de cuatro años en formulación y ejecución de proyectos de investigación relacionados con economía sectorial y estudios con enfoque de género.

\section{Mariela Pena}

Es Doctora en Antropología por la Facultad de Filosofía y Letras, Universidad de Buenos Aires (UBA) y Licenciada en Ciencias Antropológicas por la misma Universidad. Actualmente se desempeña como investigadora en el Instituto Interdisciplinario de Estudios de Género (IIEGE) de dicha casa de estudios. Ha ejercido cargos docentes a nivel universitario y ha formado parte de varios equipos de investigación. Es integrante de la Colectiva de Antropólogas Feministas (CAF). Áreas de interés: género, familia, sexualidad, infancia y parentesco.

\section{Victoria Aragón García}

Doctora en Ciencias Sociales por la Universidad de Murcia y actualmente profesora asociada de esta misma Universidad. Mis líneas de trabajo se han centrado en temas relacionados con Agua, Medioambiente, Género y Opinión pública, dentro de las cuales he participado en varios proyectos de investigacion a nivel nacional e internacional.

\section{Núria Calafell Sala}

Doctora en Teoría de la Literatura y Literatura Comparada y Licenciada en Filología Hispánica por la Universidad Autónoma de Barcelona, actualmente es Investigadora Asistente del Centro de Investigaciones y Estudios sobre Cultura y Sociedad, perteneciente al CONICET y a la 
Universidad Nacional de Córdoba, donde desarrolla un proyecto de investigación sobre la crítica como sabotaje aplicada a las manifestaciones socioculturales de Latinoamérica, en especial de Argentina.

\section{Raúl Carpio Freire}

Profesor de la Facultad de Economía y Ciencias Empresariales de la Universidad Espíritu Santo - Ecuador. Magíster en Administración de Empresas por ESPAE - ESPOL. Estudiante doctoral de Administración Estratégica de Empresas en CENTRUM - PUCP

\section{Katherine Valdiviezo Cantos}

Estudiante de la Facultad de Economía y Ciencias Empresariales de la Universidad Espíritu Santo - Ecuador. Carrera: Ingeniería comercial. Actualmente en el penúltimo año de la carrera.

\section{Tatiana Hidalgo-Marí}

Doctora por la Universidad de Alicante (2013), Magister en Comunicación e Industrias Creativas (2012) y Licenciada en Publicidad y RRPP (2007). Sus principales líneas de investigación se centran en la imagen de la mujer en la Industrias Culturales, con especial interés en la publicidad, el cine y la televisión. Miembro del grupo de investigación "Industrias culturales hoy: producción, difusión, gestión y consumo de productos culturales en la era de la información" (IIICCXXXI) de la Universidad de Alicante. Ha obtenido en dos ocasiones becas a la investigación del Centro de Estudios de la Mujer de la Universidad de Alicante y recibió el premio Bancaja-UA a la Creatividad en la Investigación (2009). En el ámbito profesional desempeña funciones como Responsable de Marketing y Comunicación en una multinacional del sector energético, actividad que compagina con el desarrollo de cursos de formación en el ámbito de la comunicación y colaboraciones editoriales.

\section{Julita Elemí Hernández}

Licenciada por la Universidad Nacional Autónoma de México y la Universidad de Texas, donde obtuvo su Doctorado. Actualmente es profesora titular de la Universidad Juárez Autónoma de Tabasco. Autora de publicaciones nacionales e internacionales, y de ponencias presentadas en congresos nacionales e internacionales. 


\section{Renán García Falconi}

Doctor en Psicología, Profesor investigador de la Universidad Juárez Autónoma de Tabasco (UJAT), México. Ha colaborado en proyectos de investigación, financiados por Fondos Mixtos y Fondos Sectoriales de CONACYT, sobre violencia de género, derechos humanos de mujeres indígenas y obesidad en niños de primaría en Tabasco, México. Ha recibido financiamiento de CONACYT para tres proyectos de investigación sobre suicidio en Tabasco y de la UJAT para 5 proyectos sobre la familia en Tabasco, calidad de vida. Ha publicado 41 artículos de investigación en revistas y memorias nacionales y extrajeras en temas relacionados con la calidad de vida, violencia de pareja, ejercicio de los derechos humanos, suicidio, entre otros. Ha participado como ponente en 51 congresos y reuniones estatales, nacionales e internacionales.

\section{Tamara Álvarez Robles}

PDI en formación de la Universidad de León. Máster en Asesoría Jurídica de Empresas por la Universidad de León. Grado en Gestión y Administración Pública por la Universidad de Oviedo. Miembro del Proyecto de Innovación Docente de la Universidad de León “Atención a la diversidad en la Universidad: Estrategias de identificación apoyo y seguimiento de colectivos con necesidades especiales, en particular, alumnos con discapacidad". Ponencia en el Congreso Internacional de Constitucionalistas (Oslo, 2014) "The migration theory: Immigration vs. Social Integration”.

\section{Leila Martina Passerino}

Licenciada en Comunicación Social por la Universidad Nacional de Entre Ríos (FCEDUUNER). Doctoranda en Ciencias Sociales, beca CONICET con sede en el Instituto de Investigaciones Gino Germani de la Universidad de Buenos Aires (IIGG- FCS-UBA). Docente en la carrera de Comunicación Social (FCEDU-UNER). Integra equipos de investigación en ambas Universidades.

\section{Noelia Soledad Trupa}

Socióloga por la Universidad de Buenos Aires (FCS-UBA). Magíster en Sociología de la Cultura y Análisis Cultural (IDAES-UNSAM). Doctoranda en Ciencias Sociales, beca 
CONICET con sede en el Instituto de Investigaciones Gino Germani (IIGG- FCSUBA). Integra equipos de investigación en la Facultad de Ciencias Sociales de la UBA.

\section{Julieta Evangelina Cano}

Magister en estudios interdisciplinares de género, Universidad de Salamanca, España. Diplomada en Género, políticas y participación, Universidad de General Sarmiento, Argentina. Docente y Secretaria de la Especialización para el abordaje de las violencias interpersonales y de género, UNLP. Becaria de doctorado del Instituto de Cultura Jurídica de la Universidad Nacional de La Plata.

\section{Dhayana Carolina Fernández Matos}

Abogada, Licenciada en Ciencias Políticas y Administrativas, Magíster en Ciencia Política y de la Administración (Universidad Autónoma de Barcelona), Magistra en Género, Identidad y Ciudadanía (Universidad de Cádiz) y Magíster en Derechos Humanos, Estado de Derecho y Democracia en Iberoamérica (Universidad de Alcalá). Fundadora de los Estudios de Género de la Escuela Nacional de la Magistratura.

\section{Diosey Ramón Lugo-Morin}

Doctor en Ciencias en Estrategias para el Desarrollo Agrícola Regional por el Colegio de Postgraduados, México. Postdoctorado por el Centro de Estudios Avanzados de la Universidad Nacional de Córdoba, Argentina. Ingeniero Agrónomo por la Universidad Central de Venezuela, Venezuela. Actividad profesional: Consultor Internacional en Adaptación al Cambio Climático y Profesor-Tutor en el Programa de Doctorado en Sostenibilidad de la Universidad Europea de Energía y Medio Ambiente. Conferencista y ponente en congresos internacionales en México, Venezuela, Ecuador, Portugal, Argentina. Miembro del Sistema Nacional de Investigadores de México.

\section{Berenice Torres-Cuapa}

Maestría en Administración Educativa por la Universidad la Salle Benavente, México. Licenciatura en Educación Media en el área de las Ciencias Sociales por la Normal Superior del Estado de Puebla, México. Especialización en competencias docentes en la sociedad del conocimiento por la Universidad Autónoma de Madrid, España. Actividad Profesional: 
Docente en la Secretaría de Educación Pública del Estado de Puebla en México. Línea de investigación: Competencias Docentes y Equidad de género.

\section{Manuela Avilés Hernández}

Doctora en Sociología por la Universidad de Alicante y profesora en el Departamento de Sociología y Trabajo Social de la Universidad de Murcia. Su principal área de interés es la Sociología de la Familia. En este ámbito investiga aspectos relacionados con las formas familiares emergentes en la sociedad española.

\section{Arancha Sánchez Manzanares}

Graduada en Trabajo Social por la Universidad de Murcia. Su línea de investigación es la Salud y el uso de nuevas técnicas en este ámbito. Colabora en la integración y promoción social de personas con enfermedades raras o síndrome de Down.

\section{Gabriela Moriana Mateo}

Doctora, Socióloga y Trabajadora Social. Directora del Máster Universitario en Género y Políticas de Igualdad y profesora del Departamento de Trabajo Social y Servicios Sociales de la Universitat de València. Sus principales líneas de investigación son la violencia contra las mujeres y la exclusión social desde la perspectiva de género.

\section{Libertad Jiménez Almirante}

Investigadora adscrita al Centro de Estudos Internacionais do Instituto Universitário de Lisboa (CEI-IUL). Doctora en Dirección de Empresas y Sociología y Master en Investigación en C.C. Sociales por la Univiversidad de Extremadura; Master en Cooperación y Desarrollo Internacional por la Universidad del País Vasco; Licenciada en Psicología por la Universidad Pontificia de Salamanca y Posgraduada en Estudios Africanos por el Instituto Universitario de Lisboa. Ha trabajado como técnica de proyectos de desarrollo en el ámbito de las ONGD y como investigadora pre-doctoral en la Universidad de Extremadura.

\section{Roxana Sosa Sánchez}

Licenciada en Ciencias Políticas y Sociología por la Universidad de Educación a Distancia (UNED), año 1993. Se doctoró por la Universidad de Oviedo, Asturias, España, en el 2005. 
Sus temas de investigación se centran en torno a la sociología del arte y de la cultura perspectiva de género.

\section{María Reyes Ferrer}

Licenciada en Filología Inglesa por la Universidad de Murcia y Filología Italiana por la Universidad de Salamanca, obtuve el título de Doctora en el 2014 en la Universidad de Sevilla, línea de investigación "mujer, escritura y comunicación”. Actualmente trabajo como Profesora Asociada en el Departemento de Filología Francesa, área de italiano y colaboro con el grupo de investigación "Escritoras y Escrituras". 